\title{
Analysis plan
}

\begin{abstract}
The purpose with this project is to evaluate if a job-matching intervention, based on formal as well as informal skills, can improve labour market opportunities for new arrivals in Sweden. The control intervention consists of regular services provided by the Swedish Employment Service (PES) which is more oriented towards preparatory courses and providing basic education, and when matching occurs, it primarily considers job seekers' formal skills. Job search assistance (JSA) has been found to be an efficient tool to reduce unemployment (Card et al., 2010, 2017). Recent evidence suggest that job-matching and vacancy referrals are important mechanisms behind the effect (Cheung et al., 2019). However, matching is normally based on formal competences and therefore not available for job-seekers lacking education and relevant labour market experience. Such job-seekers are often classified as non-matchable and assigned to preparatory programs for which there are no evidence of increasing transition to employment.
\end{abstract}

\section{Interventions}

The intervention studied in this project is a Job search assistance (JSA) intervention designed to offer job search and matching assistance to job seekers who have been in Sweden only for a short period of time and where some seekers lack education and relevant job experience. The intervention is therefore designed to include both formal as well as informal competences in the matching process.

The program starts with a broad mapping of the job seekers' formal and informal skills, desired professions and their requests for working hours. The match is then supposed to take place by surveying potential employers based on the characteristics identified among the job seekers. The match between the job seeker and the potential employer is then supported by PES throughout the process of the job-seeker's entry into the labour market, both during the interview, and with follow-ups when needed.

The control intervention consists of regular services provided by the Swedish Employment Service (PES) which is more oriented towards preparatory courses and providing basic education, and when matching occurs, it primarily considers job seekers' formal skills.

\section{Primary outcomes}

a. Non-subsidized and subsidized regular work

b. Regular education (education that is not part of the by interventions operated by the Swedish employment office)

c. How fast participants go to regular work (days until a regular work is obtained)

d. Quality of the work measured by the monthly full-time wage received

e. Intermediate and long-term labour market outcomes such as annual and monthly labour earnings, monthly full-time equivalent wages, hiring- and separations, occupational and industry affiliation

f. The probability of leaving Sweden 


\section{Heterogenous effects}

In this study we will analyses heterogenous effects using the machine learning algorithms ass suggested by for example Chernozhukov et al. (2018) and Wager and Athey (forthcoming).

\section{Experimental design}

The target group are newly arrived immigrants in need of protection (as refugee, subsidiary protection, and including their family members), who have had residence permit up to four years. All job seekers from the target group are included in the target population and explicitly made eligible for selection to the study. The project is constructed as a randomized controlled trial (RCT) which enables us to evaluate the effects of the program. Hence, study participants are randomized into either the JSA program or the control group. Study participation is conditional on informed consent about the general purpose of the study.

Until randomization, all participants only receive general information where specific information on each intervention is not mentioned. In the next step, those randomized into the treatment group receive information on the availability of the JSA program. Job seekers randomized into the control group continue the meeting with the caseworker and continue to have access to all services at the PES that would have been available to them outside of the study.

- Randomization done in office by a random generator

- Individual level randomization within clusters consisting of combinations of 16 geographical locations at the PES, gender and months during the period March 2019 to April 2021. This, in total 832 clusters $(2 \times 26 \times 16)$

- The cluster level consists of 16 times 24 location-months cells.

- Sample size 6,500-9,000

- Swedish Ethical Review Authority 